\title{
Intraoperative Neurological Monitoring During Neck Dissection for Esophageal Cancer With Aberrant Subclavian Artery
}

\author{
HIROYUKI KITAGAWA, JUN IWABU, KEIICHIRO YOKOTA, \\ TSUTOMU NAMIKAWA and KAZUHIRO HANAZAKI
}

Department of Surgery, Kochi Medical School, Nankoku, Japan

\begin{abstract}
We report a case of esophageal cancer with a non-recurrent inferior laryngeal nerve associated with aberrant right subclavian artery that was treated by neck dissection using intraoperative neurological monitoring followed by thoracoscopic esophagectomy. A 76-year-old man had dysphagia. Endoscopy revealed thoracic esophageal cancer, and computed tomography revealed the presence of an aberrant right subclavian artery between the esophagus and vertebrae. We performed neck dissection followed by thoracoscopic esophagectomy. During the neck dissection, we confirmed a non-recurrent inferior laryngeal nerve through intraoperative neurological monitoring. No postoperative complications were observed, and the patient was discharged 19 days after surgery. We recommend using intraoperative neurological monitoring to avoid injury to the non-recurrent inferior laryngeal nerve associated with the aberrant right subclavian artery.
\end{abstract}

The presence of an aberrant right subclavian artery (ARSA), a vessel anomaly reported in approximately 0.6$1.3 \%$ of patients (1), should be considered important during the treatment of esophageal squamous cell carcinoma (ESCC) because of its association with the non-recurrent inferior laryngeal nerve (NRILN). Herein, we report the case of a patient with ESCC accompanied by NRILN associated with ARSA, who was treated with neck dissection; intraoperative neurological monitoring (IONM) was used to avoid injury to NRILN, followed by thoracoscopic esophagectomy (TSE).

Correspondence to: Tsutomu Namikawa, Department of Surgery, Kochi Medical School, Kohasu-Okocho, Nankoku, Kochi, 7838505, Japan. Tel: +81 888802370, Fax: +81 888802371, e-mail: tsutomun@kochi-u.ac.jp

Key Words: Intraoperative neurological monitoring, aberrant right subclavian artery, esophageal cancer, non-recurrent inferior laryngeal nerve.

\section{Case Report}

A 76-year-old man had dysphagia. Endoscopy revealed a type-0-IIa+IIc tumor, located $25 \mathrm{~cm}$ to $35 \mathrm{~cm}$ from the teeth. Biopsy revealed a squamous cell carcinoma. Computed tomography (CT) revealed no evidence of metastasis, although it detected an ARSA. We confirmed that the ARSA originated from the aortic arch. The patient was diagnosed with stage I thoracic ESCC with NRILN, and we decided to perform neck dissection using IONM to avoid injury to the NRILN, followed by TSE.

For IONM, the NIM-Response 3.0 (Medtronics Inc., Minneapolis, MN, USA) neurological monitoring system was adopted. During the neck dissection, we visually detected the right vagus nerve and several nerves along its course (Figure 1). Thereafter, we stimulated them to confirm the NRILN. When NRILN was correctly stimulated (Figure 2), an alarm rang, and the amplitude was visualized on the monitor (Figure 3); stimulation of other tissue did not activate the alarm, and the amplitude was not visualized. After neck dissection with preservation of NRILN, we performed TSE with the patient in the prone position. After TSE, a gastric tube was created, and post-mediastinum route reconstruction with circular stapler anastomosis was performed at the neck. No postoperative complications were observed, and the patient was discharged 19 days after surgery.

\section{Discussion}

ARSA is a vessel anomaly reported in approximately 0.6$1.3 \%$ of patients (1). When CT reveals an ARSA, the presence of NRILN should be considered (2). Few reports are available on the usefulness of IONM to detect the NRILN associated with ARSA during esophageal surgery.

We previously reported a case of thoracic ESCC with ARSA and NRILN, and recommended neck dissection prior to TSE to detect the ARSA (3). However, the detection of the ARSA depended on visual confirmation according to the Toniato classification, which classified the pattern of NRILN 


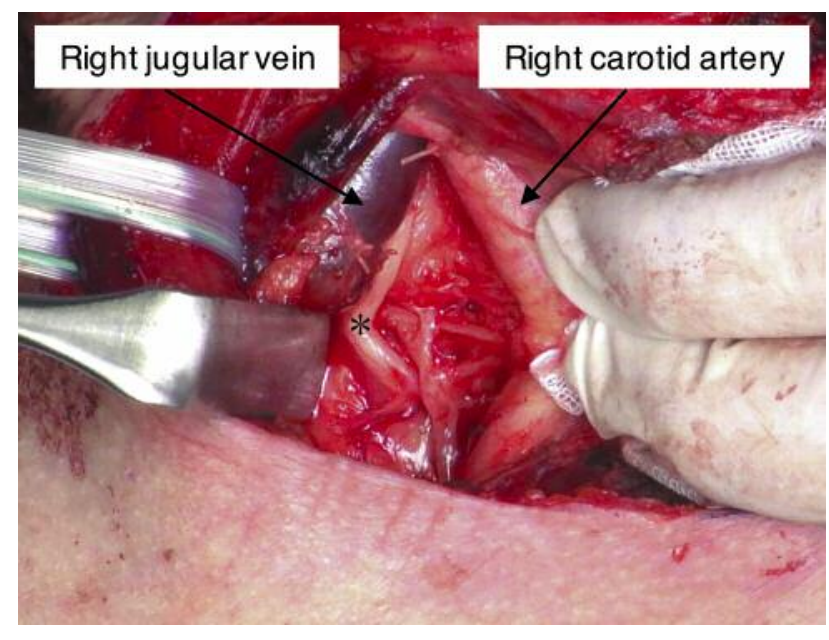

Figure 1. Operative view during right neck dissection. During the neck dissection, the right vagus nerve (asterisk) and several nerves were seen; however, the visual detection of the non-recurrent inferior laryngeal nerve was difficult.

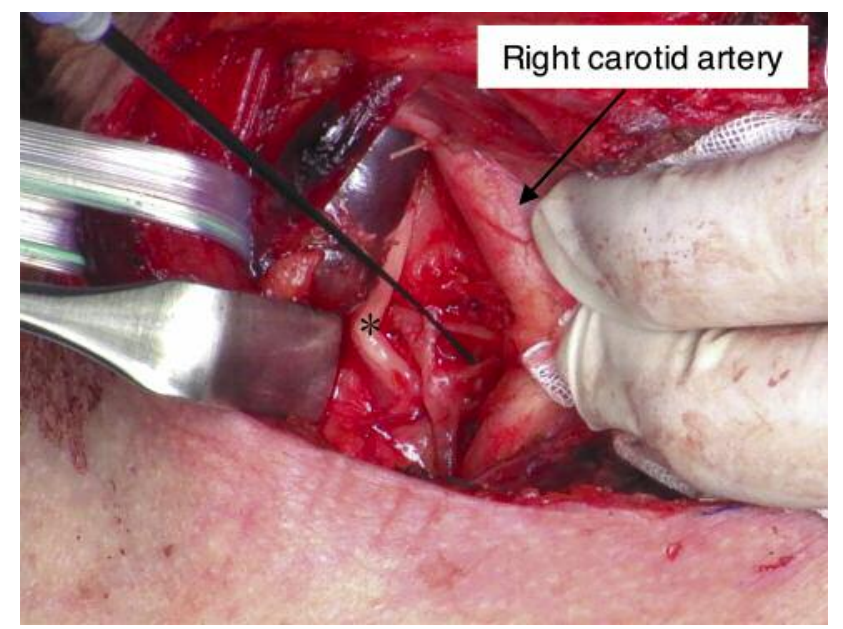

Figure 2. Intermittent stimulation of the non-recurrent inferior laryngeal nerve during neck dissection. Asterisk shows the right vagus nerve.

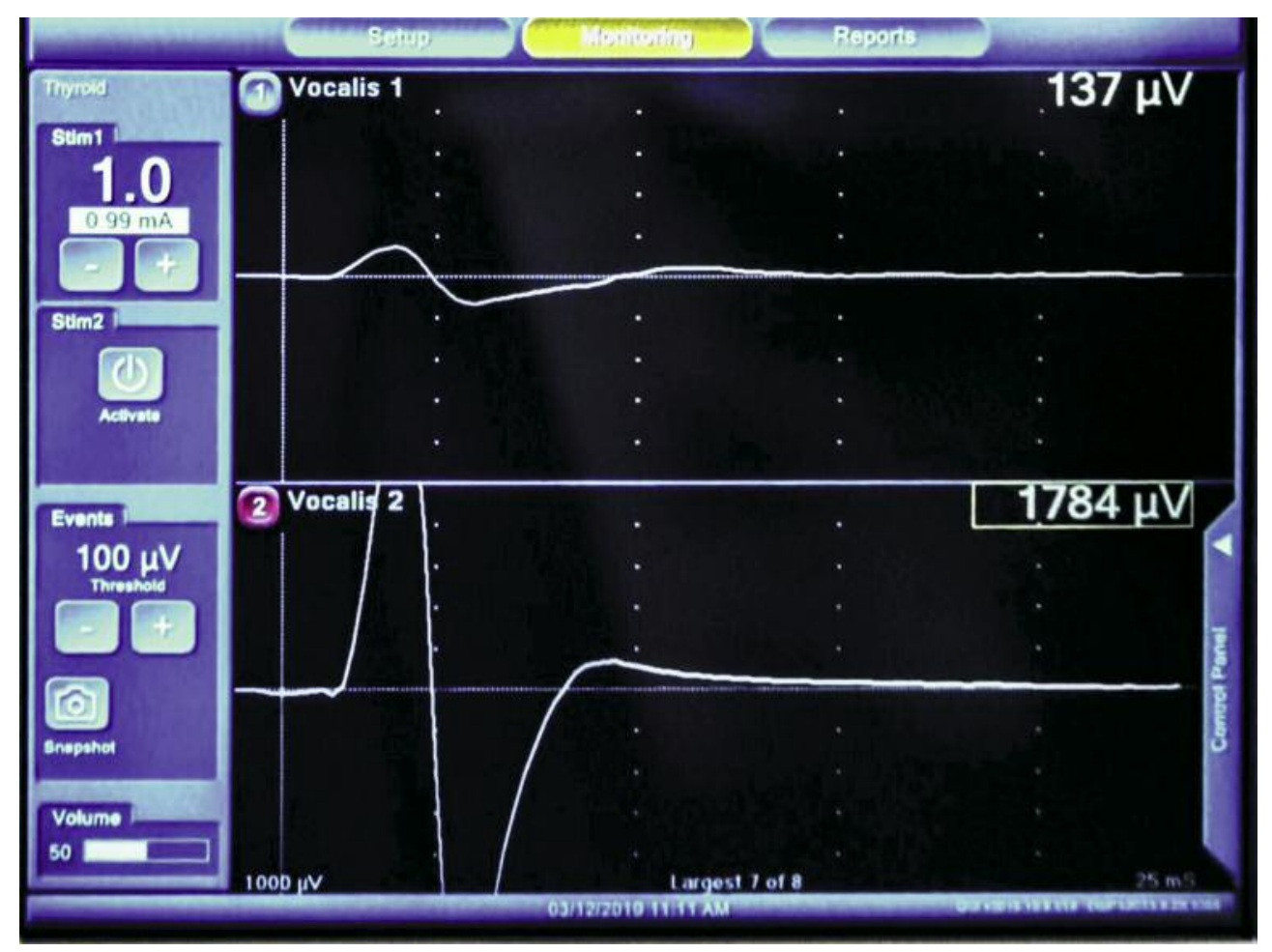

Figure 3. Intraoperative neurological monitoring. The upper and lower screens show the amplitude of the left and right vocal cords, respectively. On stimulation of the non-recurrent inferior laryngeal nerve, a strong amplitude was seen and the alarm was activated.

into the following three types: type 1 , arising from the vagus nerve and running directly into the larynx; type $2 \mathrm{a}$, running together with the inferior thyroid artery; and type $2 b$, running under the inferior thyroid artery (4). In the present study, visual confirmation of NRILN was difficult because several nerves were present along the course of the vagus nerve, and the use of IONM was effective to confirm and preserve the true NRILN. 
Two methods for IONM, namely the continuous and intermittent stimulation methods, are available. Some researchers reported the utility of intraoperative continuous vagus nerve stimulation to identify the site of recurrent nerve injury during TSE (5). However, intermittent stimulation was useful to detect the NRILN during neck dissection by the sounding of an alarm and visual (amplitude) information on stimulation of the NRILN. However, upon stimulation of other tissue; no audio-visual information was relayed.

\section{Conclusion}

We recommend using IONM during neck dissection prior to TSE in cases of thoracic ESCC with ARSA and NRILN.

\section{Conflicts of Interest}

The Authors declare no conflicts of interest related to this study.

\section{Authors' Contributions}

HK and TN drafted the manuscript and all other authors participated in the design of the study. All Authors were involved in editing the manuscript and approved the final text of the manuscript.

\section{Acknowledgements}

The Authors would like to thank Editage (www.editage.jp) for English language editing.

\section{References}

1 Henry JF, Audiffret J, Denizot A and Plan M: The nonrecurrent inferior laryngeal nerve: review of 33 cases, including two on the left side. Surgery 104(6): 977-984, 1988. PMID: 3057672.

2 Watanabe A, Kawabori S, Osanai H, Taniguchi $M$ and Hosokawa M: Preoperative computed tomography diagnosis of non-recurrent inferior laryngeal nerve. Laryngoscope 111(10): 1756-1759, 2001. PMID: 11801940. DOI: 10.1097/00005537200110000-00017

3 Kitagawa H, Namikawa T and Hanazaki K: Neck dissection and thoracoscopic esophagectomy in esophageal cancer with aberrant subclavian artery. Anticancer Res 37(7): 3787-3790, 2017. PMID: 28668875. DOI: 10.21873/anticanres.11754

4 Toniato A, Mazzarotto R, Piotto A, Bernante P, Pagetta C and Pelizzo MR: Identification of the nonrecurrent laryngeal nerve during thyroid surgery: 20-year experience. World J Surg 28(7): 659-661, 2004. PMID: 15175898. DOI: 10.1007/s00268-0047197-7

5 Tsang RK and Law S: Adaptation of continuous intraoperative vagus nerve stimulation for monitoring of recurrent laryngeal nerve during minimally invasive esophagectomy. World J Surg 40(1): 137-41, 2016. PMID: 26464154. DOI: 10.1007/s00268$015-3265-4$

Received April 19, 2019

Revised May 6, 2019

Accepted May 7, 2019 